\title{
Entomofauna Associada a Galhos de Acacia mangium Willd. Roletados por Oncideres saga (Dalman) (Coleoptera: Cerambycidae)
}

\author{
Gláucia Cordeiro $^{1 \bowtie}{ }^{\bowtie}$, Norivaldo dos Anjos ${ }^{1} \&$ Acacio Geraldo de Carvalho²
}

Agência de Fomento: CAPES. 1. Universidade Federal de Viçosa, Viçosa-MG, e-mail: glaucordeiro@gmail.com (Autor para correspondência ${ }^{\bowtie}$ ), nanjos@ufv.br 2. Universidade Federal Rural do Rio de Janeiro, Seropédica-RJ, e-mail: acacio@ufrrj.br.

EntomoBrasilis 3 (1): 22-24 (2010)

Resumo. O estudo da entomofauna associada aos galhos e fustes roletados por Oncideres saga (Dalman) é importante para conhecermos seus possíveis inimigos naturais. Portanto, este trabalho teve como objetivo registrar os insetos associados aos galhos e fustes de Acacia mangium Willd. roletados por O. saga, em Coimbra-MG. Galhos e fustes roletados de A. mangium foram coletados de janeiro a abril de 2007. Este material foi vistoriado, armazenados em sacolas, feitas com tela plástica, e mantidos em sala com condições controladas $\left(25,4 \pm 0,3^{\circ} \mathrm{C}\right.$ e $\left.66,7 \pm 1,4 \%\right)$. Constatou-se a presença de uma espécie, não determinada, de Scolytidae e a emergência de quatro espécies de Cerambycidae: Engyum quadrinotatum Thomsom; Eburodacrys sexmaculata (Olivier); Achryson surinamum (Linnaeus) e Neoclytus pusillus (Laporte \& Gory). Isto posto, fica evidenciada a necessidade de estudos com o objetivo de verificar qual o comportamento destes insetos em relação ao serrador $O$. saga.

Palavra- chave: Coleoptera, Inseto, Serrador.

\section{Insects associated with branches of Acacia mangium Willd. girdled by Oncideres saga (Dalman)}

\section{(Coleoptera: Cerambycidae)}

Abstract. The study of the insects associated with branches and stems girdled by Oncideres saga (Dalman) is important to know its possible natural enemies. Therefore, these work had the objective of register the insects associated with branches and stems girdled of Acacia mangium Willd. by this twig girdler beetle, in Coimbra, state of Minas Gerais, Brazil. Stems and branches of A. mangium were collected in January/2007 to April/2007. This material has been inspected, stored in plastic bags, and kept in a room with controlled conditions $\left(25.4 \pm 0.3^{\circ} \mathrm{C}\right.$ and $\left.66.7 \pm 1.4 \%\right)$. It was noted the presence of a non-determined species of Scolytidae and the emergence of four species of Cerambycidae: Engyum quadrinotatum Thomsom; Eburodacrys sexmaculata (Olivier); Achryson surinamum (Linnaeus) and Neoclytus pusillus (Laporte \& Gory). It can be concluded that studies are needed with the objective of verify the behavior of these insects in relation with twig girdler $O$. saga.

Keywords: Coleoptera, Insect, Twig girdler

$A$ cacia mangium Willd. (Fabaceae: Mimosoideae) é conhecida popularmente por Acácia-mangium, Mangium, Acácia ou Acácia-australiana (ARCO-VERDE 2002; LORENZI et al. 2003). Esta espécie possui origem australiana e é uma essência florestal fixadora de nitrogênio no solo, que se adapta a terrenos degradados e bem drenados (NATIONAL RESEARCH COUNCIL 1983). Por causa destas características, esta espécie é usada na recuperação de solos degradados (SCHIAVO \& MARTINS 2003). Esta espécie apresenta rápido crescimento e alta produção madeireira podendo assim ser utilizada para substituir a madeira de espécies nativas na produção de lenha (SOUZA et al. 2004). A madeira de Acácia-mangium é usada na produção de moirões, construção civil, como quebraventos (BALIEIRO et al. 2004), na produção de carvão, chapa de fibra de média densidade (MDF), aglomerados e compensados (SCHIAVO \& MARTINS 2003). As flores desta espécie são melíferas (BALIEIRO et al. 2004) e o néctar extrafloral pode ser usado por abelhas do gênero Apis para a produção de mel (BARBOSA 2002).

Entre os insetos daninhos às árvores de Acácia-mangium os coleópteros conhecidos como serradores, também, têm causado freqüentes e expressivos prejuízos aos acaciais plantados no Sudeste do Brasil (COUTINHO et al. 1998). Os serradores são insetos pertencentes à ordem Coleoptera, família Cerambycidae e subfamília Lamiinae, que roletam ramos e ponteiros de árvores em pleno vigor (LIMA 1955). De acordo com FERREIRA (1975), roletar significa "cortar em redor de" e, segundo CORDEIRO (2008), é exatamente o que o serrador faz, ele corta ao redor do galho ou fuste, o qual acaba caindo devido ao próprio peso ou por causa da ação do vento. Dentro dessas partes roletadas, além do serrador, são mencionados insetos pertencentes à ordem Coleoptera, e Hymenoptera se desenvolvendo (BUCK 1957 e 1962; KIRCH 1983; DI IORIO 1994 e 1996; WITECK NETO \& LINK 1997; MAGISTRALI et al. 2008).

O conhecimento da entomofauna dos ecossistemas florestais tem sua importância no que se refere aos danos provocados pelos seus diferentes segmentos (WITECK NETO \& LINK 1997) e aos possíveis inimigos naturais de determinada praga. Assim, este trabalho teve como objetivo registrar os insetos associados aos galhos de A. mangium roletados por Oncideres saga (Dalman), no Município de Coimbra-MG.

Vistorias em um plantio de $A$. mangium, localizado em Coimbra-MG, com presença pré-determinada de $O$. saga foram realizadas com a finalidade de coletar galhos e fustes roletados por este serrador (Figura 1). O material coletado foi vistoriado, para verificar se apresentavam algum sinal da presença de outros insetos e em seguida armazenados em sacolas, feitas com tela plástica $(64 \times 35 \mathrm{~cm})$. Inspeções semanais foram realizadas para constatar possíveis associações com outros insetos. Os galhos foram mantidos em sala com temperatura e umidade relativa do ar igual a $25,4 \pm 0,3^{\circ} \mathrm{C}$ e $66,7 \pm 1,4 \%$, respectivamente, e umedecidos periodicamente. Os insetos emergidos foram 
coletados, montados e enviados ao Dr. Miguel A. Monné, para determinação específica.

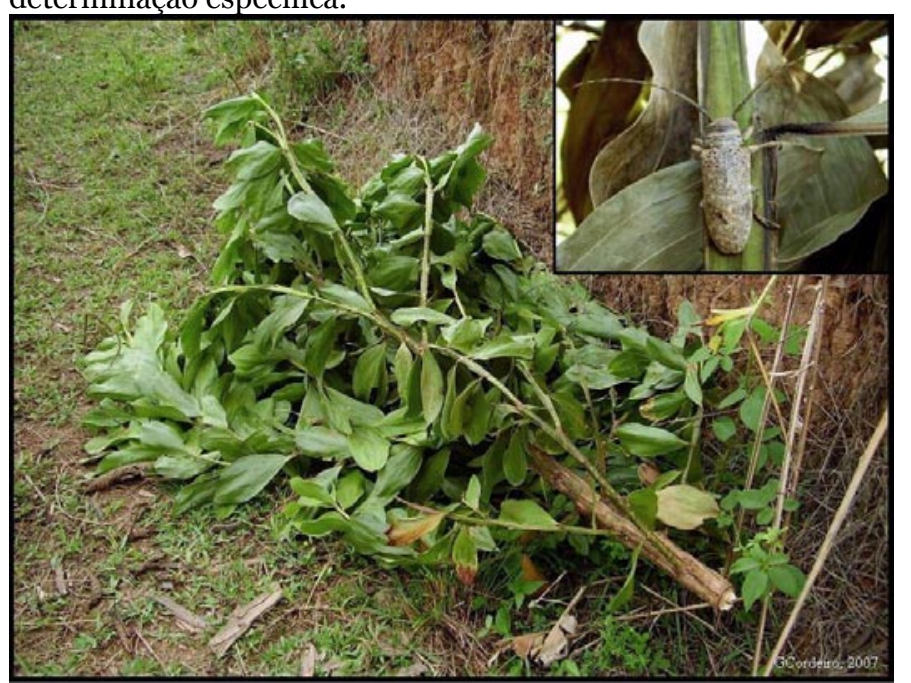

Figura 1: Fuste de Acacia mangium Willd. roletado por Oncideres saga (Dalman, 1823). Em detalhe, adulto de O. saga. Coimbra, MG. 2007.

Foram coletados sete fustes e dois galhos laterais roletados por O. saga. Ao vistoriar o material coletado, constatouse a presença de besouros da família Scolytidae junto a seis ovos de $O$. saga. Todos os ovos estavam com uma de suas extremidades aberta e sem embrião (Figura 2A), sendo que a metade desses ovos apresentava coloração escura (Figura $2 \mathrm{~B}$ ), provavelmente devido a fungo. Como esta ocorrência foi registrada no último fuste de $A$. mangium, roletado e recolhido, não foram possíveis à verificação de novas ocorrências e nem a avaliação exata do comportamento deste escolitídeo em relação aos ovos de O. saga.

Os insetos emergidos pertencem à família Cerambycidae, subfamília Cerambycinae e foram determinados como, Engyum quadrinotatum Thomsom, Eburodacrys sexmaculata (Olivier), Achryson surinamum (Linnaeus) e Neoclytus pusillus (Laporte \& Gory) (Figura 3). BAUCKE (1962) relata as espécies $A$. surinamum, N. pusillus e Eburodacrys sexmaculata Lam., broqueando galhos, troncos e ramos de Acacia mearnsii De Willd. Recentemente MAGISTRALI et al. (2008) constatou a presença de $A$. surinamum associada a galhos de $A$. mearnsii roletados por $O$. saga. Os cerambicídeos relatados neste trabalho já foram relatados desenvolvendo-se dentro de galhos roletados por serradores de outras espécies de Oncideres (BUCK, 1957; DI IORIO, 1994; DI IORIO, 1996; WITECK NETO \& LINK, 1997). Exemplares destes cerambicídeos emergidos foram incorporados na coleção do Museu Regional de Entomologia UFVB.
Diante do exposto, fica evidenciada a necessidade de estudos com o objetivo de verificar qual o comportamento do escolitídeo em relação aos ovos de $O$. saga. Além de verificar se os adultos dos cerambicídeos estão predando os ovos de $O$. saga ou até mesmo se as larvas deles estão competindo com as deste serrador; ou se eles estão apenas aproveitando o galho e o fuste caído, para completarem seu ciclo, uma vez que estes não são capazes de realizar o roletamento, mas são atraídos por madeira recém-abatida no início de fermentação.

\section{AGRADECIMENTO}

Ao Dr. Miguel A. Monné, da Universidade Federal do Rio de Janeiro, na determinação das espécies de Cerambycidae. A CAPES pelo incentivo à pesquisa através do fornecimento de bolsa.

\section{REFERÊNCIAS}

Arco-Verde, M.F., 2002. Potencialidades e usos da Acacia mangium Willd. no estado de Roraima. Boa Vista: Embrapa Roraima, 18 p. (Embrapa Roraima. Documentos, 6).

Balieiro, F.C., L.E.Dias, A.A. Franco, E.F.C. Campello \& S.M. Faria, 2004. Acúmulo de nutrientes na parte aérea, na serapilheira acumulada sobre o solo e decomposição de filódios de Acacia mangium Willd.. Ciência Florestal, 14: 59-65.

Barbosa, R.I. 2002. Florestamento dos sistemas de vegetação aberta (savanas/cerrados) de Roraima por espécies exóticas. Disponível em: <http://agroeco.inpa.gov. br/reinaldo/RIBarbosa ProdCient Usu Visitantes/ 2002AcaciaTemasDiscussao CEMAT.pdf.> [30 jan. 2008].

Baucke, O., 1962. A inseto-fauna da acácia negra no Rio Grande do Sul: Biologia e controle às pragas mais importantes. Porto Alegre, Secretaria da Agricultura/Secção de Informações e Publicidade Agrícola, $32 \mathrm{p}$.

Buck, P., 1957. Insetos criados em galhos cortados. Iheringia, 47.

Cordeiro, G. 2008. Aspectos biológicos de Oncideres saga (Dalman) (Coleoptera: Cerambycidae) e efeitos de seus danos em Acacia mangium Willd. 82 f. Dissertação. (Mestrado em Entomologia) - Universidade Federal de Viçosa.

Coutinho, C.L., A.C., Carvalho, E.S. Oliveira \& B.G.A.Veiga, 1998. Oncideres saga (Dalman, 1823) (Coleoptera: Cerambycidae) e a arborização urbana em Seropédica, RJ. Floresta e Ambiente, 5: 50-54.

Di Iorio, O.R., 1994. Cerambycidae y outros Coleoptera emergidos de ramas cortadas por Oncideres germari (Lamiinae: Onciderini) em el norte argentino. Revista de Biologia Tropical, 42: 649-661.

Di Iorio, O.R., 1996. Cerambycidae y otros Coleoptera emergidos

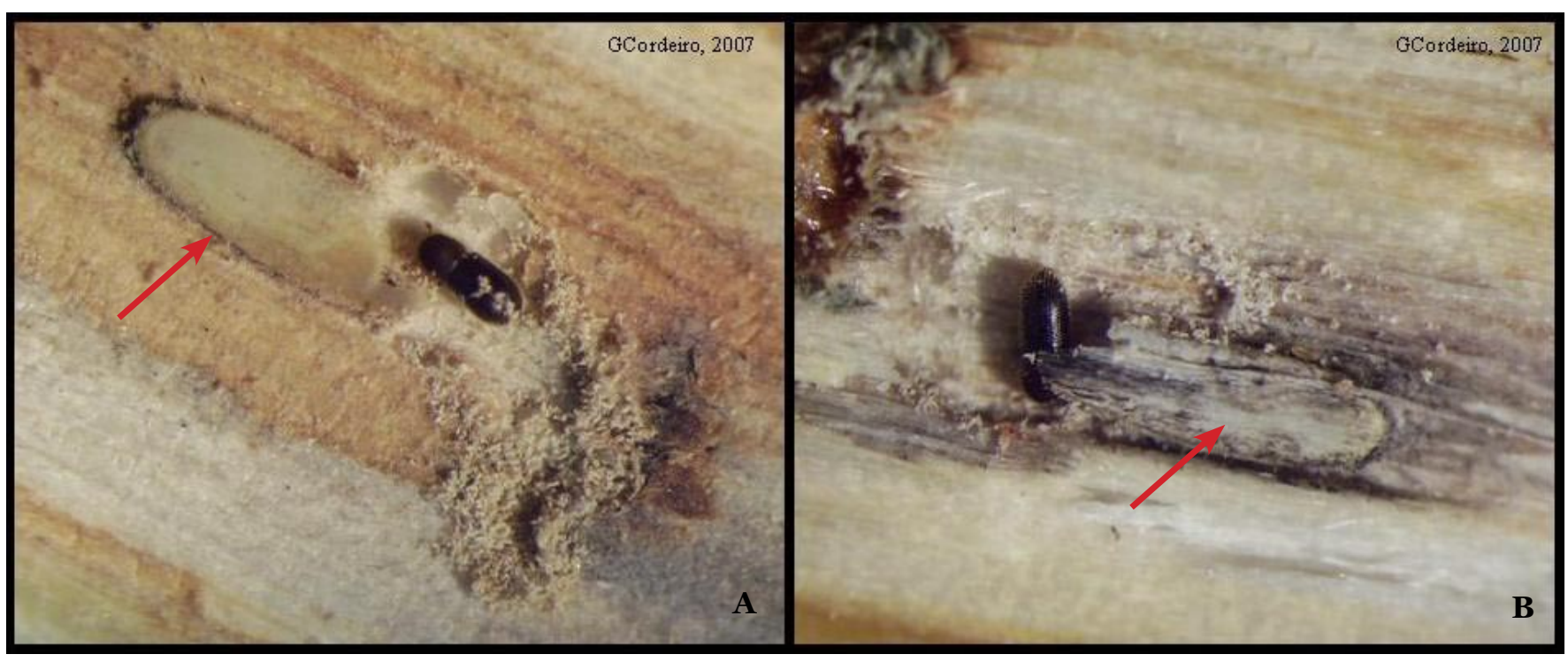

Figura 2. Escolitídeo junto ao ovo de Oncideres saga (Dalman), em fuste de Acacia mangium Willd.. (A) Ovo com uma de suas extremidades aberta e sem embrião; (B) Ovo com coloração escura. Coimbra, MG. 2007. (Seta indica o ovo) 


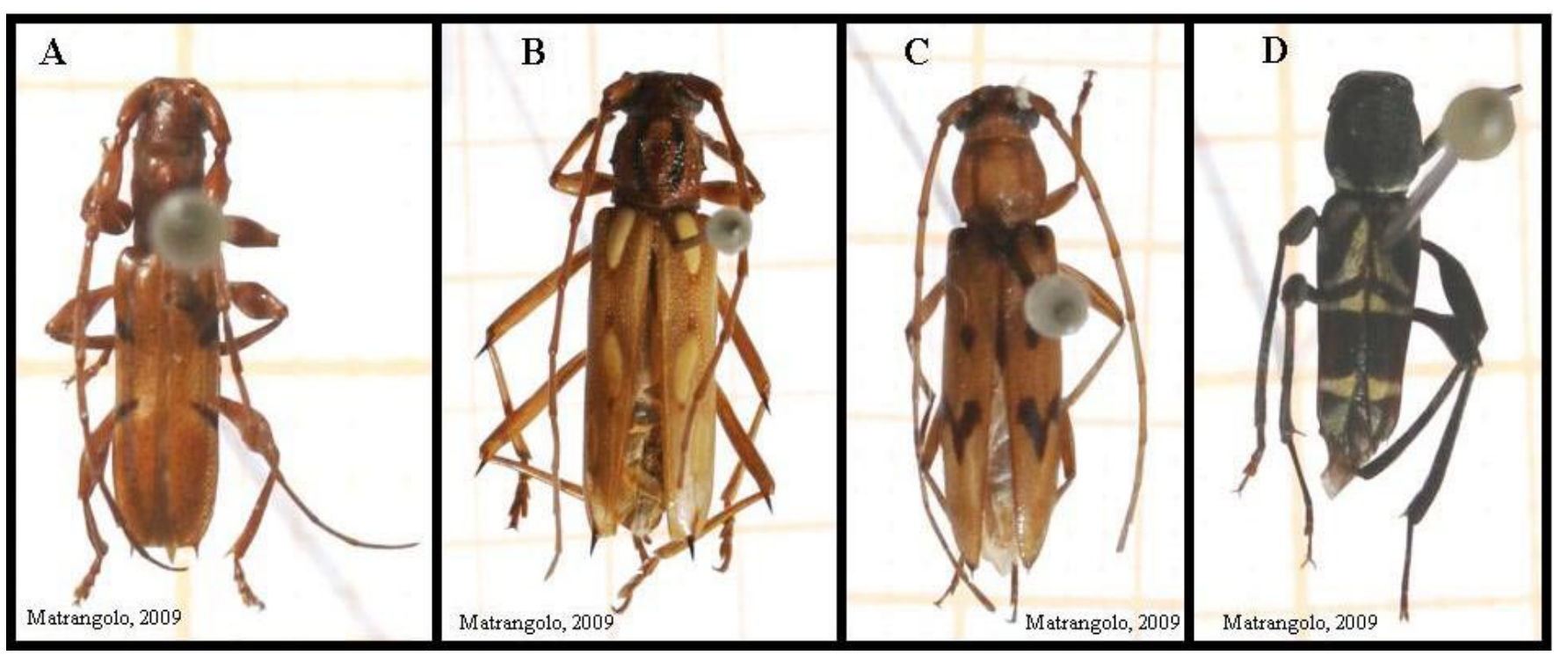

Figura 3. Cerambicídeos emergidos dos galhos e fustes de Acacia mangium Willd. roletados por Oncideres saga (Dalman): (A) Engyum quadrinotatum Thomsom; (B) Eburodacrys sexmaculata (Olivier); (C) Achryson surinamum (Linnaeus) e (D) Neoclytus pusillus (Laporte \& Gory). Coimbra, MG. 2007.

de ramas cortadas por Oncideres germari (Lamiinae: Onciderini) en el Norte Argentino. Revista de Biologia Tropical, 44: 551-565.

Ferreira, A.B.H. 1975. Novo Dicionário da Língua Portuguesa. Rio de Janeiro: Editora Nova Fronteira S. A. 1499 p. 1a Edição (10a Impressão).

Kirch, E. 1983. Estudo dos inimigos naturais de Oncideres impluviata (Germar, 1824) em Mimosa Scabrella Benth. 65p. Dissertação (Mestrado em Ciências) - Universidade Federal do Paraná.

Lima, A.C., 1955. Insetos do Brasil: Coleópteros. Rio de Janeiro, Escola Nacional de Agronomia, 9o Tomo, 3a Parte. 289p. (Série Didática, 11).

Lorenzi, H., H.M. Souza, M.A.V. Torres \& L.B. Bacher, 2003. Árvores exóticas no Brasil: madeiras, ornamentações e aromáticas. Nova Odessa: Instituto Plantarum, 368p.

Magistrali, I.C., P.R. Magistrali, J. De Nadai \& A.A. Goellner, 2008. Parâmetros biológicos de Oncideres saga (Dalman, 1823) (Coleoptera: Cerambycidae) em Acacia mearnsii De Willd.. Revista Trópica - Ciências Agrárias e Biológicas, 310.

National Research Council, 1983. Mangium and other fast-

\section{Como citar este artigo:}

Cordeiro, G., N. dos Anjos \& A.G. Carvalho, 2010. Entomofauna Associada a Galhos de Acacia mangium Willd. Roletados por Oncideres saga (Dalman) (Coleoptera: Cerambycidae). EntomoBrasilis, 3(1): 23-24. www.periodico.ebras.bio.br/ojs growing acacias for the humid tropics. Washington, Nacional Academy Press, 62 p.

Schiavo, J.A. \& M.A.Martins, 2003. Produção de mudas de Acácia colonizadas com micorrizas e rizóbio em diferentes recipientes. Pesquisa Agropecuária Brasileira, 38: 173-178.

Souza, C.R., L.M.B. Rossi, C.P. Azevedo \& R.M.B. Lima, 2004. Comportamento da Acacia mangium e de clones de Eucalyptus grandis $\mathrm{x}$ E. urophylla em plantios experimentais na Amazônia Central. Scientia Forestalis, 95-101.

Witeck Neto, L. \& D. Link, 1997. Cerambycidae associados a Lauraceae, na região central do Rio Grande do Sul, Brasil. Ciência Florestal, 7: 33-39.

Recebido em: 14/09/2009

Aceito em: 14/12/2009
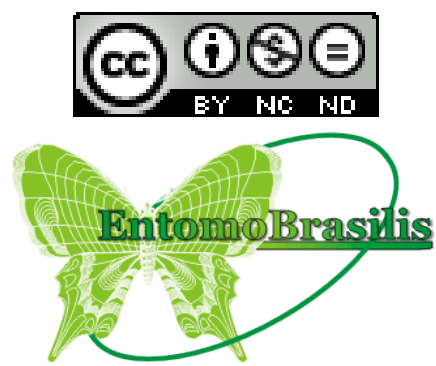